\title{
Splines, lattice points, and (arithmetic) matroids
}

\author{
Matthias Lenz"k
}

Mathematical Institute, University of Oxford, Oxford, United Kingdom

\begin{abstract}
Let $X$ be a $(d \times N)$-matrix. We consider the variable polytope $\Pi_{X}(u)=\{w \geq 0: X w=u\}$. It is known that the function $T_{X}$ that assigns to a parameter $u \in \mathbb{R}^{N}$ the volume of the polytope $\Pi_{X}(u)$ is piecewise polynomial. Formulas of Khovanskii-Pukhlikov and Brion-Vergne imply that the number of lattice points in $\Pi_{X}(u)$ can be obtained by applying a certain differential operator to the function $T_{X}$. In this extended abstract we slightly improve the formulas of Khovanskii-Pukhlikov and Brion-Vergne and we study the space of differential operators that are relevant for $T_{X}$ (i.e. operators that do not annihilate $T_{X}$ ) and the space of nice differential operators (i.e. operators that leave $T_{X}$ continuous). These two spaces are finite-dimensional homogeneous vector spaces and their Hilbert series are evaluations of the Tutte polynomial of the (arithmetic) matroid defined by $X$.

Résumé. Soit $X$ une matrice $(d \times N)$. Nous considérons le polytope variable $\Pi_{X}(u)=\{w \geq 0: X w=u\}$. Il est connu que la fonction $T_{X}$ qui attribue à un paramètre $u$ le volume du polytope $\Pi_{X}(u)$ est polynomiale par morceaux. Des formules de Khovanskii-Pukhlikov et de Brion-Vergne impliquent que le nombre de points de réseau dans $\Pi_{X}(u)$ peut être obtenu en appliquant un certain opérateur différentiel à la fonction $T_{X}$. Dans ce résumé élargi nous améliorons un peu les formules de Khovanskii-Pukhlikov et de Brion-Vergne et nous étudions l'espace d'opérateurs différentiels qui sont importants pour $T_{X}$ (c'est-à-dire les opérateurs qui n'annulent pas $T_{X}$ ) et l'espace d'opérateurs différentiels bons (c'est-à-dire les opérateurs qui laissent $T_{X}$ continue). Ces deux espaces sont espaces vectoriels homogène de dimension finie et leurs séries de Hilbert sont des évaluations du polynôme de Tutte du matroïde (arithmétique) défini par $X$.
\end{abstract}

Keywords: lattice polytope, box spline, vector partition function, Todd operator, (arithmetic) matroid, zonotopal algebra

\section{Introduction}

Box splines and multivariate splines measure the volume of certain variable polytopes. Since vector partition functions measure the number of integral points in these polytopes, they can be seen as discrete versions of the spline functions. Splines and vector partition functions have recently received a lot of attention by researchers in various fields including approximation theory, algebra, combinatorics, and representation theory. A standard reference from the approximation theory point of view is the book [9]

*Email: lenz@maths.ox.ac.uk. The author was supported by a Junior Research Fellowship of Merton College (Oxford). 
by de Boor, Höllig, and Riemenschneider. The combinatorial and algebraic aspects are stressed in the book [10] by De Concini and Procesi.

Khovanskii and Pukhlikov proved a remarkable formula that relates the volume and the number of integer points in a smooth polytope [15]. The connection is made via Todd operators, i.e. differential operators of type $\frac{\partial_{x}}{1-e^{\partial_{x}}}$. The formula is closely related to the Hirzebruch-Riemann-Roch Theorem for smooth projective toric varieties (see [6, Chapter 13]). Brion and Vergne have extended the KhovaniskiiPukhlikov formula to arbitrary rational polytopes [5].

In this extended abstract we will slightly improve the formulas of Khovanskii-Pukhlikov and BrionVergne. Instead of the Todd operator, which is a formal power series with an infinite number of terms, we use a polynomial that is obtained as a truncation of a shifted version of the Todd operator. It has the advantage that it leaves the spline continuous.

We also study spaces of partial differential operators with periodic coefficients that are relevant for $T_{X}$ (i.e. operators that do not annihilate $T_{X}$ ) and spaces of nice differential operators (i.e. operators that leave $T_{X}$ continuous). The theory becomes considerably simpler if one assumes that the matrix $X$ that defines the objects is unimodular. In this case, the spaces of differential operators are the so-called $\mathcal{P}$-spaces [2, 14], i. e. they have constant coefficients and they are related with matroid theory via the Tutte polynomial. In the general case there is a connection with arithmetic matroids and the arithmetic Tutte polynomial.

Our results in the unimodular case are explained in more detail and proven in the preprint [18]. The results in Sections 5 and 6 are new and not published yet. Various related result have been presented at FPSAC in recent years, e.g. on $\mathcal{P}$-spaces and power ideals [2], arithmetic matroids and their Tutte polynomials [7, 20], and on box splines [19].

Organisation of the article. The remainder of this article is organised as follows: in Section 2 we introduce our notation and the objects that we study. In Section 3 we state the results in the case where the matrix $X$ is unimodular. In Section 4 we define deletion and contraction and briefly explain how one can prove the results in Section 3 . In Section 5 we discuss the general case and in Section 6 we explain some connections between the general case and arithmetic matroids. In Section 7 we give some examples.

\section{Preliminaries}

In this section we introduce our notation and the objects that we study. The notation is similar to the one used in [10]. We fix a $d$-dimensional real vector space $U$ and a lattice $\Lambda \subseteq U$. Let $X=\left(x_{1}, \ldots, x_{N}\right) \subseteq \Lambda$ be a finite list of vectors that spans $U$. The list $X$ is called unimodular with respect to $\Lambda$ if and only if every basis for $U$ that can be selected from $X$ is also a lattice basis for $\Lambda$. Note that $X$ can be identified with a linear map $X: \mathbb{R}^{N} \rightarrow U$. Let $u \in U$. We define the variable polytopes

$$
\Pi_{X}(u):=\left\{w \in \mathbb{R}_{\geq 0}^{N}: X w=u\right\} \quad \text { and } \quad \Pi_{X}^{1}(u):=\Pi_{X}(u) \cap[0 ; 1]^{N} .
$$

Note that any convex polytope can be written in the form $\Pi_{X}(u)$ for suitable $X$ and $u$. The dimension of these two polytopes is at most $N-d$. We define the

$$
\begin{aligned}
\text { vector partition function } \mathcal{T}_{X}(u) & :=\left|\Pi_{X}(u) \cap \mathbb{Z}^{N}\right| \\
\text { the box spline } B_{X}(u) & :=\operatorname{det}\left(X X^{T}\right)^{-1 / 2} \operatorname{vol}_{N-d} \Pi_{X}^{1}(u),
\end{aligned}
$$




$$
\text { and the multivariate spline } T_{X}(u):=\operatorname{det}\left(X X^{T}\right)^{-1 / 2} \operatorname{vol}_{N-d} \Pi_{X}(u) .
$$

Note that we have to assume that 0 is not contained in the convex hull of $X$ in order for $T_{X}$ and $\mathcal{T}_{X}$ to be well-defined. Otherwise, $\Pi_{X}(u)$ may be unbounded. The zonotope $Z(X)$ is defined as

$$
Z(X):=\left\{\sum_{i=1}^{N} \lambda_{i} x_{i}: 0 \leq \lambda_{i} \leq 1\right\}=X \cdot[0,1]^{N}
$$

We denote its set of interior lattice points by $\mathcal{Z}_{-}(X):=\operatorname{int}(Z(X)) \cap \Lambda$. The symmetric algebra over $U$ is denoted by $\operatorname{Sym}(U)$. We fix a basis $s_{1}, \ldots, s_{d}$ for the lattice $\Lambda$. This makes it possible to identify $\Lambda$ with $\mathbb{Z}^{d}, U$ with $\mathbb{R}^{d}, \operatorname{Sym}(U)$ with the polynomial $\operatorname{ring} \mathbb{R}\left[s_{1}, \ldots, s_{d}\right]$, and $X$ with a $(d \times N)$-matrix. Then $X$ is unimodular if and only if every non-singular $(d \times d)$-submatrix of this matrix has determinant 1 or -1 . The base-free setup is more convenient when working with quotients of vector spaces.

We denote the dual vector space by $V=U^{*}$ and we fix a basis $t_{1}, \ldots, t_{d}$ that is dual to the basis for $U$. An element of $\operatorname{Sym}(U)$ can be seen as a differential operator on $\operatorname{Sym}(V)$, i. e. $\operatorname{Sym}(U) \cong \mathbb{R}\left[s_{1}, \ldots, s_{d}\right] \cong$ $\mathbb{R}\left[\frac{\partial}{\partial t_{1}}, \ldots, \frac{\partial}{\partial t_{d}}\right]$. For $f \in \operatorname{Sym}(U)$ and $p \in \operatorname{Sym}(V)$ we write $f(D) p$ to denote the polynomial in $\operatorname{Sym}(V)$ that is obtained when $f$ acts on $p$ as a differential operator. It is known that the box spline and the multivariate spline are piecewise polynomial. We will mostly use elements of $\operatorname{Sym}(U)$ as differential operators on their local pieces.

Note that a vector $u \in U$ defines a linear form $u \in \operatorname{Sym}(U)$. For a sublist $Y \subseteq X$, we define $p_{Y}:=\prod_{y \in Y} y$. For example, if $Y=((1,0),(1,2))$, then $p_{Y}=s_{1}\left(s_{1}+2 s_{2}\right)$. Furthermore, $p_{\emptyset}:=1$. We define the rank of a sublist $Y \subseteq X$ as the dimension of the vector space spanned by $Y$. We denote it by $\operatorname{rk}(Y)$. Now we define the

$$
\begin{aligned}
\text { central } \mathcal{P} \text {-space } \mathcal{P}(X) & :=\operatorname{span}\left\{p_{Y}: \operatorname{rk}(X \backslash Y)=\operatorname{rk}(X)\right\} \\
\text { and the internal } \mathcal{P} \text {-space } \mathcal{P}_{-}(X): & =\bigcap_{x \in X} \mathcal{P}(X \backslash x) .
\end{aligned}
$$

The space $\mathcal{P}(X)$ first appeared in approximation theory [1, 8, 12]. The space $\mathcal{P}_{-}(X)$ was introduced in [14] where it was also shown that if $X$ is unimodular then the dimension of this space is equal to $\left|\mathcal{Z}_{-}(X)\right|$ and $\operatorname{dim} \mathcal{P}(X)=\operatorname{vol} Z(X)$. More specifically, it is known [2, 14] that

$$
\operatorname{Hilb}\left(\mathcal{P}_{-}(X), q\right)=q^{|(x \in X: x \neq 0)|-d} \mathfrak{T}_{X}\left(0, q^{-1}\right) \text { and } \operatorname{Hilb}(\mathcal{P}(X), q)=q^{|(x \in X: x \neq 0)|-d \mathfrak{T}_{X}\left(1, q^{-1}\right)},
$$

where $\mathfrak{T}_{X}$ denotes the Tutte polynomial of the matroid defined by $X$ and $\operatorname{Hilb}(\bullet, q)$ denotes the Hilbert series of the graded vector space $\bullet$.

\section{Results in the unimodular case}

In this section we describe our results in the case where the list $X$ is unimodular. The splines $B_{X}$ and $T_{X}$ are piecewise polynomial. The regions of polynomiality are $d$-dimensional cones in the case of $T_{X}$ and $d$-dimensional polytopes in the case of $B_{X}$. The splines are obviously smooth in the interior of the regions of polynomiality. This is in general not the case where two regions of polynomiality overlap. The following theorem characterises the differential operators that leave the splines continuous. 
Theorem 1 ([[18]). Let $X \subseteq \Lambda \subseteq U \cong \mathbb{R}^{d}$ be a list of vectors that is unimodular and spans $U$. Then

$$
\mathcal{P}_{-}(X)=\left\{f \in \mathcal{P}(X): f(D) B_{X} \text { is a continuous function }\right\} .
$$

Note that since $B_{X}(u)=\sum_{A \subseteq X}(-1)^{|A|} T_{X}\left(u-\sum_{x \in A} x\right)$, a differential operator $f(D)$ with constant coefficients leaves $B_{X}$ continuous if and only if it leaves $T_{X}$ continuous. Theorem 1 ensures that the derivatives of $B_{X}$ that appear in the following theorem exist.

Theorem 2 ([19], conjectured in [14]). Let $X \subseteq \Lambda \subseteq U \cong \mathbb{R}^{d}$ be a list of vectors that is unimodular and spans $U$. Let $f$ be a real valued function on $\mathcal{Z}_{-}(\bar{X})$, the set of interior lattice points of the zonotope defined by $X$.

Then there exists a unique polynomial $p \in \mathcal{P}_{-}(X) \subseteq \mathbb{R}\left[s_{1}, \ldots, s_{d}\right]$, s.t. $\left.p(D) B_{X}\right|_{\mathcal{Z}_{-}(X)}=f$.

Let $z \in U$. As usual, the exponential is defined as $e^{z}:=\sum_{k \geq 0} \frac{z^{k}}{k !} \in \mathbb{R}\left[\left[s_{1}, \ldots, s_{d}\right]\right]$. We define the ( $z$-shifted) Todd operator

$$
\operatorname{Todd}(X, z):=e^{-z} \prod_{x \in X} \frac{x}{1-e^{-x}} \in \mathbb{R}\left[\left[s_{1}, \ldots, s_{d}\right]\right] .
$$

The Todd operator was introduced by Hirzebruch in the 1950s [13] and plays a fundamental role in the Hirzebruch-Riemann-Roch theorem for complex algebraic varieties. It can be expressed in terms of the Bernoulli numbers $B_{0}=1, B_{1}=-\frac{1}{2}, B_{2}=\frac{1}{6}, \ldots$ Recall that they are defined by the equation $\frac{s}{e^{s}-1}=\sum_{k \geq 0} \frac{B_{k}}{k !} s^{k}$. One should note that $e^{z} \frac{z}{e^{z}-1}=\frac{z}{1-e^{-z}}=\sum_{k \geq 0} \frac{B_{k}}{k !}(-z)^{k}$. For $z \in \mathcal{Z}_{-}(X)$ we can fix a list $S \subseteq X$ s.t. $z=\sum_{x \in S} x$. Let $T:=X \backslash S$. Then we can write the Todd operator as $\operatorname{Todd}(X, z)=\prod_{x \in S} \frac{x}{e^{x}-1} \prod_{x \in T} \frac{x}{1-e^{-x}}$.

A sublist $C \subseteq X$ is called a cocircuit if $\operatorname{rk}(X \backslash C)<\operatorname{rk}(X)$ and $C$ is inclusion minimal with this property. We consider the cocircuit ideal $\mathcal{J}(X):=\operatorname{ideal}\left\{p_{C}: C\right.$ cocircuit $\} \subseteq \operatorname{Sym}(U)$. It is known [12, 14] that $\operatorname{Sym}(U)=\mathcal{P}(X) \oplus \mathcal{J}(X)$.

Let $\psi_{X}: \mathcal{P}(X) \oplus \mathcal{J}(X) \rightarrow \mathcal{P}(X)$ denote the projection. Note that this is a graded linear map and that $\psi_{X}$ maps to zero any homogeneous polynomial whose degree is at least $N-d+1$. This implies that there is a canonical extension $\psi_{X}: \mathbb{R}\left[\left[s_{1}, \ldots, s_{d}\right]\right] \rightarrow \mathcal{P}(X)$ given by $\psi_{X}\left(\sum_{i}\left(g_{i}\right)\right):=\sum_{i} \psi_{X}\left(g_{i}\right)$, where $g_{i}$ denotes a homogeneous polynomial of degree $i$. Let

$$
f_{z}=f_{z}^{X}:=\psi_{X}(\operatorname{Todd}(X, z))
$$

Example 3. For $X=(1,1)$ we obtain $\operatorname{Todd}((1,1), 1)=\left(1+B_{1} s+\ldots\right)\left(1-B_{1} s+\ldots\right)=1+0 s+\ldots$ Hence $f_{1}^{(1,1)}=1 \in \mathcal{P}_{-}(1,1)=\mathbb{R}$. Note that $\mathcal{P}(1,1)=\operatorname{span}\{1, s\}$ and $\mathcal{J}(1,1)=$ ideal $\left\{s^{2}\right\}$.

Remark 4. It is known that for $f \in \mathcal{J}(X), f(D) B_{X}=f(D) T_{X}=0$. On the other hand, if $f \in \mathcal{P}(X)$, then $f(D) B_{X} \neq 0$ and $f(D) T_{X} \neq 0$. Hence $\mathcal{P}(X)$ can be seen as the space of relevant differential operators on $B_{X}$ and $T_{X}$ with constant coefficients.

Theorem 5 ([18]). Let $X \subseteq \Lambda \subseteq U \cong \mathbb{R}^{d}$ be a list of vectors that is unimodular and spans $U$. Let $z$ be a lattice point in the interior of the zonotope $Z(X)$.

Then $f_{z} \in \mathcal{P}_{-}(X)$, $\operatorname{Todd}(X, z)(D) B_{X}$ extends continuously on $U$, and

$$
\left.f_{z}(D) B_{X}\right|_{\Lambda}=\left.\operatorname{Todd}(X, z)(D) B_{X}\right|_{\Lambda}=\delta_{z} .
$$


Dahmen and Micchelli observed that

$$
T_{X}=B_{X} *_{d} \mathcal{T}_{X}:=\sum_{\lambda \in \Lambda} B_{X}(\cdot-\lambda) \mathcal{T}_{X}(\lambda)
$$

(cf. [10, Proposition 17.17]). The symbol $*_{d}$ stands for (semi-)discrete convolution. Using this result, the following variant of the Khovanskii-Pukhlikov formula [15] follows immediately.

Corollary 6 ([18]). Let $X \subseteq \Lambda \subseteq U \cong \mathbb{R}^{d}$ be a list of vectors that is unimodular and spans $U$. Let $u \in \Lambda$ and $z \in \mathcal{Z}_{-}(X)$. Then

$$
\left|\Pi_{X}(u-z) \cap \Lambda\right|=\mathcal{T}_{X}(u-z)=\operatorname{Todd}(X, z)(D) T_{X}(u)=f_{z}(D) T_{X}(u) .
$$

Remark 7. Theorem 5 is related to a deconvolution formula formula of De Concini, Procesi, and Vergne [11]. They showed that $\left.\operatorname{Todd}(X, 0)(D) p_{\mathfrak{c}}\right|_{\Lambda}=\delta_{0}$, where $p_{\mathfrak{c}}$ denotes a local piece of the box spline $B_{X}$, which is the case $z=0$ in Theorem 14 below. As $B_{X}$ is not everywhere sufficiently often differentiable, $\operatorname{Todd}(X, 0)$ can only be applied to its local pieces.

One can deduce from [11, Remark 3.15] that $\operatorname{Todd}(X, z)(D) B_{X}$ can be extended continuously if $z \in \mathcal{Z}_{-}(X)$. It is also not difficult to show that $\operatorname{Todd}(X, z)(D) B_{X}=f_{z}(D) B_{X}$ and that multiplying the Todd operator by $e^{-x}$ corresponds to translating $\operatorname{Todd}(X, z)(D) B_{X}$ by $x$. The novelty of Theorem 5 is that the operator $f_{z}$ for $z \in \mathcal{Z}_{-}(X)$ is shorter than the original Todd operator operator (cf. Example 28, i. e. it is contained in $\mathcal{P}_{-}(X)$.

Remark 8. Theorem 14 below implies that if $X$ is unimodular and $z$ is an arbitrary lattice point in the zonotope $Z(X)$, then

$$
\left|\Pi_{X}(u-z) \cap \Lambda\right|=\mathcal{T}_{X}(u-z)=\operatorname{Todd}(X, z)(D) p_{\Omega}(u)=f_{z}(D) p_{\Omega}(u),
$$

where $p_{\Omega}$ denotes a suitable local piece of $T_{X}$. The original Khovanskii-Pukhlikov formula is the case $z=0$ in (15). For more information on this formula, see Vergne's survey article on integral points in polytopes [21]. An explanation of the Khovanskii-Pukhlikov formula that is easy to read is contained in the book by Beck and Robins [4, Chapter 10].

Corollary 9 ([18]). Let $X \subseteq \Lambda \subseteq U \cong \mathbb{R}^{d}$ be a list of vectors that is unimodular and spans $U$. Then $\sum_{z \in \mathcal{Z}_{-}(X)} B_{X}(z) f_{z}=1$. This implies formula $[13)$.

Proposition 10 ([12]). Let $X \subseteq U \cong \mathbb{R}^{d}$ be a finite list of vectors that spans $U$. A basis for $\mathcal{P}(X)$ is given by $\mathcal{B}(X):=\left\{Q_{B}: B \in \mathbb{B}(X)\right\}$, where $Q_{B}:=p_{X \backslash(B \cup E(B))}$ and $E(B)$ denotes the set of externally active elements in $X$ with respect to the basis $B$.

Various other generalised $\mathcal{P}$-spaces also have a canonical basis [14, 17]. Up to now, no general construction for a basis of the internal space $\mathcal{P}_{-}(X)$ was known (cf. [3, 14, 16]). The polynomials $f_{z}$ form such a basis.

Corollary 11 ([18]). Let $X \subseteq \Lambda \subseteq U \cong \mathbb{R}^{d}$ be a list of vectors that is unimodular and spans $U$. Then $\left\{f_{z}: z \in \mathcal{Z}_{-}(X)\right\}$ is a basis for $\mathcal{P}_{-}(X)$.

We also obtain a new basis for the central space $\mathcal{P}(X)$. Let $w \in U$ be a short affine regular vector, i.e. a vector whose Euclidian length is very small that is not contained in a hyperplane generated by a sublist of $X$. Let $\mathcal{Z}(X, w):=(Z(X)-w) \cap \Lambda$. It is known that $\operatorname{dim} \mathcal{P}(X)=|\mathcal{Z}(X, w)|=\operatorname{vol}(Z(X))$ [14].

Corollary 12 ([18]). Let $X \subseteq \Lambda \subseteq U \cong \mathbb{R}^{d}$ be a list of vectors that is unimodular and spans $U$. Then $\left\{f_{z}: z \in \mathcal{Z}(X, w)\right\}$ is a basis for $\overline{\mathcal{P}}(X)$. 


\section{Deletion-contraction, limits, and proof idea}

In this section we define deletion and contraction and discuss the deletion-contraction proof of Theorem 5 . We also describe a way to make sense of derivatives of piecewise polynomial functions via limits that is used in the proof.

Deletion-contraction. Let $x \in X$. We call the list $X \backslash x$ the deletion of $x$. The image of $X \backslash x$ under the canonical projection $\pi_{x}: U \rightarrow U / \operatorname{span}(x)=: U / x$ is called the contraction of $x$. It is denoted by $X / x$.

The projection $\pi_{x}$ induces a map $\operatorname{Sym}(U) \rightarrow \operatorname{Sym}(U / x)$ that we will also denote by $\pi_{x}$. If we identify $\operatorname{Sym}(U)$ with the polynomial ring $\mathbb{R}\left[s_{1}, \ldots, s_{d}\right]$ and $x=s_{d}$, then $\pi_{x}$ is the map from $\mathbb{R}\left[s_{1}, \ldots, s_{d}\right]$ to $\mathbb{R}\left[s_{1}, \ldots, s_{d-1}\right]$ that sends $s_{d}$ to zero and $s_{1}, \ldots, s_{d-1}$ to themselves. Note that since $X$ is unimodular, $\Lambda / x \subseteq U / x$ is a lattice for every $x \in X$ and $X / x$ is unimodular with respect to this lattice.

All structures studied this article behave nicely under deletion and contraction if $X$ is unimodular. Namely:

(i) The box spline satisfies $D_{x} B_{X}=\nabla_{x} B_{X \backslash x}:=B_{X \backslash x}-B_{X \backslash x}(\cdot-x)$ (e.g. [9]) and $B_{X / x}(\bar{u})=$ $\sum_{\lambda \in \mathbb{Z}} B_{X}(u+\lambda x)([19])$.

(ii) There is a canonical bijection $\mathcal{Z}_{-}(X) \backslash \mathcal{Z}_{-}(X \backslash x) \rightarrow \mathcal{Z}_{-}(X / x)$ (e.g. [19]).

(iii) The equations $x f_{z}^{X \backslash x}=f_{z}^{X}-f_{z+x}^{X}$ and $\pi_{x}\left(f_{z}^{X}\right)=f_{\bar{z}}^{X / x}$ hold ([18]).

(iv) The following sequence is exact: $0 \rightarrow \mathcal{P}_{-}(X \backslash x) \stackrel{\cdot x}{\longrightarrow} \mathcal{P}_{-}(X) \stackrel{\pi_{x}}{\longrightarrow} \mathcal{P}_{-}(X / x) \rightarrow 0$ and the same sequence is also exact for $\mathcal{P}(X)([2])$.

For every $z \in \mathcal{Z}_{-}(X)$ there is a unique polynomial $q_{z}^{X}=q_{z} \in \mathcal{P}_{-}(X)$ s.t. $\left.q_{z}(D) B_{X}\right|_{\Lambda}=\delta_{z}$ by Theorem 2. In order to prove Theorem 5 it is sufficient to show that $f_{z}=q_{z}$. In fact, $q_{z}$ and $f_{z}$ behave in the same way under deletion and contraction, i. e. they both satisfy the equalities in (iii) above. Unfortunately, it is not obvious that $f_{z} \in \mathcal{P}_{-}(X)$.

Therefore, we have to make a detour. Since $\mathcal{P}_{-}(X)$ is in general not spanned by polynomials of type $p_{Y}$ for some $Y \subseteq X$ (cf. [3]), it is quite difficult to handle this space. The space $\mathcal{P}(X)$ on the other hand has a basis which is very convenient for deletion-contraction (cf. Proposition 10 ). In the larger space $\mathcal{P}(X)$ we are able to prove by deletion-contraction that $f_{z}=q_{z}$.

Differentiating piecewise polynomial functions and limits. In this paragraph we state an extension of Theorem 5 to differential operators in $\mathcal{P}(X)$. In general for $f \in \mathcal{P}(X),\left.f(D) B_{X}\right|_{\Lambda}$ may not be well-defined. However, it is possible to define it as a certain limit.

Definition 13. Let $H$ by a hyperplane spanned by a sublist $Y \subseteq X$. A shift of such a hyperplane by a vector in the lattice $\Lambda$ is called an affine admissible hyperplane. An alcove is a connected component of the complement of the union of all affine admissible hyperplanes

A vector $w \in U$ is called affine regular, if it is not contained in any affine admissible hyperplane. We call $w$ short affine regular if it is affine regular and it is so short that it is contained in an alcove whose closure contains the origin.

Note that on the closure of each alcove $\mathfrak{c}, B_{X}$ agrees with a polynomial $p_{\mathfrak{c}}$. For example, the six triangles in the figure in Example 27 are the alcoves where $B_{X}$ agrees with a non-zero polynomial. The regions of polynomiality of $T_{X}$ are full-dimensional convex cones that are called big cells.

Fix a short affine regular vector $w \in U$. Let $u \in \Lambda$. Let $\mathfrak{c} \subseteq U$ be an alcove s.t. $u$ and $u+\varepsilon w$ are contained in its closure for some small $\varepsilon>0$ and let $p_{\mathfrak{c}}$ be the polynomial that agrees with $B_{X}$ on the 
closure of $\mathfrak{c}$. For a differential operator $f(D)$ we define

$$
\lim _{w} f\left(D_{\mathrm{pw}}\right) B_{X}(u):=f(D) p_{\mathfrak{c}}(u)
$$

(pw stands for piecewise). For $T_{X}, \lim _{w} f\left(D_{\mathrm{pw}}\right) T_{X}$ can be defined in the same way. More information on this construction can be found in [11] where it was introduced. Note that the limit can be dropped if $f(D) B_{X}$ is continuous at $u$. Otherwise, the limit is important: note for example that $\lim _{w} B_{(1)}(0)$ is either 1 or 0 depending on whether $w$ is positive or negative.

Recall that $\mathcal{Z}(X, w):=(Z(X)-w) \cap \Lambda$.

Theorem 14 ([18]). Let $X \subseteq \Lambda \subseteq U \cong \mathbb{R}^{d}$ be a list of vectors that is unimodular and spans $U$. Let $w$ be a short affine regular and let $z \in \mathcal{Z}(X, w)$. Then

$$
\left.\lim _{w} f_{z}\left(D_{\mathrm{pw}}\right) B_{X}\right|_{\Lambda}=\left.\lim _{w} \operatorname{Todd}(X, z)\left(D_{\mathrm{pw}}\right) B_{X}\right|_{\Lambda}=\delta_{z} .
$$

\section{Results in the general case}

In this section we discuss the case of a list $X \subseteq \Lambda$ that is not necessarily unimodular. In this case everything becomes a bit more complicated. In the unimodular case, both $\mathcal{T}_{X}$ and $T_{X}$ are piecewise polynomial. In the non-unimodular case, $T_{X}$ is still piecewise polynomial but the vector partition function $\mathcal{T}_{X}$ is piecewise quasipolynomial. A function $f$ defined on a lattice $\Lambda$ is quasipolynomial if there exists a sublattice $\Lambda^{0} \subseteq \Lambda$ s.t. $f$ restricted to each coset of $\Lambda^{0}$ is equal to (the restriction of) a polynomial. Hence, the differential operator that turns $T_{X}$ into $\mathcal{T}_{X}$ must turn polynomials into quasipolynomials, i.e. in general it has periodic (non-constant) coefficients. These periodic coefficients are related to the vertices of the toric arrangement defined by $X$.

We first introduce toric arrangements and the space $\widetilde{\mathcal{P}}(X)$, a space of differential operators with periodic coefficients, before discussing analogues of the results in Section 3

Toric arrangements are arrangements of hypersurfaces on a torus. We denote that dual lattice of $\Lambda$ by $\Gamma:=\{v \in V: v(\lambda) \in \mathbb{Z}$ for all $\lambda \in \Lambda\}$. Note that if we identify $U$ and $V$ with $\mathbb{R}^{d}$ and a basis for $\Lambda$ is given by the columns of a $(d \times d)$-matrix, then the rows of the inverse of this matrix form a basis for $\Gamma$. Recall that a vector $x \in U$ defines a hyperplane $H_{x}=\{v \in V: v(x)=0\}$. The set $H_{x}^{p}=\{v \in V: v(x) \in \mathbb{Z}\}$ is a periodic arrangement of countably many shifts of the hyperplane $H_{x}$. Note that $\gamma(x) \in \mathbb{Z}$ for all $\gamma \in \Gamma$ if $x \in \Lambda$. This implies that for all $x \in X, \Gamma$ acts on $H_{x}^{p}$ by translation. The quotient $H_{x}^{t}:=H_{x}^{p} / \Gamma=\{v \in V / \Gamma: v(x)=0\}$ is a (possibly disconnected) hypersurface in the torus $V / \Gamma \cong(\mathbb{R} / \mathbb{Z})^{d}$.

Definition 15 (toric arrangements). Let $X \subseteq \Lambda \subseteq U \cong \mathbb{R}^{d}$ be a finite list of vectors that spans $U$. The set $\left\{H_{x}^{t}: x \in X\right\}$ is called the toric arrangement defined by $X$.

The set $\bigcup\left(\bigcap_{x \in B} H_{x}^{t}\right)$ where the union runs over all bases $B \subseteq X$ is a finite set. It is called the set of vertices of the toric arrangement and denoted by $\mathcal{V}(X)$.

See Figure 2(a) for an example. Let $\phi \in \mathcal{V}(X)$. We define a sublist $X \supseteq X_{\phi}:=(x \in X: \phi(x)=0)$. This is the maximal sublist of $X$ such that $\phi \in \bigcap_{x \in X_{\phi}} H_{x}^{t}$. Furthermore, we define the exponential $e_{\phi}: U \rightarrow \mathbb{C}$ by $e_{\phi}(u):=\exp (2 \pi i \phi(u))$.

The periodic coefficient analogue of the central $\mathcal{P}$-space is the

$$
\text { periodic central } \mathcal{P} \text {-space } \widetilde{\mathcal{P}}(X):=\bigoplus_{\phi \in \mathcal{V}(X)} e_{\phi} p_{X \backslash X_{\phi}} \mathcal{P}\left(X_{\phi}\right) \subseteq \bigoplus_{\phi \in \mathcal{V}(X)} e_{\phi} \operatorname{Sym}(U)
$$


Definition 16 (Periodic Todd operator). Let $X \subseteq U \cong \mathbb{R}^{d}$ be a finite list of vectors and let $z \in U$. Then we define the ( $z$-shifted) periodic Todd operator

$$
\widetilde{\operatorname{Todd}}(X, z):=e^{-z} \sum_{\phi \in \mathcal{V}(X)} e_{\phi} \prod_{x \in X} \frac{x}{1-e_{\phi}(-x) e^{-x}} .
$$

Note that if the list $X$ is unimodular, then $\mathcal{V}(X)=\{0\}$. This implies $\widetilde{\operatorname{Todd}}(X, z)=\operatorname{Todd}(X, z)$ and $\widetilde{\mathcal{P}}(X)=\mathcal{P}(X)$.

Theorem 17 (Brion-Vergne, [5]). Let $X \subseteq \Lambda \subseteq U \cong \mathbb{R}^{d}$ be a finite list of vectors that spans $U$. Let $u \in \operatorname{cone}(X)$ and let $w \in U$ be a short affine regular vector s. $t . u+w \in \operatorname{cone}(X)$. Then

$$
\lim _{w} \underset{\operatorname{Todd}}{\widetilde{O}}(X, 0)\left(D_{\mathrm{pw}}\right) T_{X}(u)=\mathcal{T}_{X}(u)
$$

Recall that we defined earlier a projection map $\psi_{X}: \mathbb{R}\left[\left[s_{1}, \ldots, s_{d}\right]\right] \rightarrow \mathcal{P}(X)$. Now we require a projection $\widetilde{\psi}_{X}: \bigoplus_{\phi \in \mathcal{V}(X)} e_{\phi} \mathbb{R}\left[\left[s_{1}, \ldots, s_{d}\right]\right] \rightarrow \widetilde{\mathcal{P}}(X)$.

Let $f \in \bigoplus_{\phi \in \mathcal{V}(X)} e_{\phi} \mathbb{R}\left[\left[s_{1}, \ldots, s_{d}\right]\right]$. Then $f$ can be written uniquely as $f=\sum_{\phi \in \mathcal{V}(X)} e_{\phi} f_{\phi}$ for some $f_{\phi} \in \mathbb{R}\left[\left[s_{1}, \ldots, s_{d}\right]\right]$. We define

$$
\widetilde{\psi}_{X}(f):=\sum_{\phi} e_{\phi} \psi_{X}\left(f_{\phi}\right) \quad \text { and } \quad \tilde{f}_{z}:=\widetilde{\psi}_{X}(\widetilde{\operatorname{Todd}}(X, z)) .
$$

We are able to deduce the following result from Theorem 17

Theorem 18. Let $X \subseteq \Lambda \subseteq U \cong \mathbb{R}^{d}$ be a finite list of vectors that spans $U$. If $z \in \mathcal{Z}_{-}(X)$, then $\tilde{f}_{z}(D) T_{X}$ is continuous in $\Lambda$. Hence, the following formula holds:

$$
\left.\tilde{f}_{z}(D) T_{X}\right|_{\Lambda}=\mathcal{T}_{X}(\cdot-z) .
$$

Note that $\left.\tilde{f}_{z}(D) T_{X}\right|_{\Lambda}$ is only continuous in $\Lambda$ and will usually be discontinuous at the non-lattice points where two regions of polynomiality overlap (cf. Figure 2(b)).

Corollary 19. Let $X \subseteq \Lambda \subseteq U$ be a finite list of vectors that spans $U$. Then $\sum_{z \in \mathcal{Z}_{-}(X)} B_{X}(z) \tilde{f}_{z}=1$.

We define the internal periodic $\mathcal{P}$-space $\widetilde{\mathcal{P}}_{-}(X):=\operatorname{span}\left\{\tilde{f}_{z}: z \in \mathcal{Z}_{-}(X)\right\}$. The following result is a periodic analogue of Theorem 1

Theorem 20. Let $X \subseteq \Lambda \subseteq U \cong \mathbb{R}^{d}$ be a list of vectors that spans $U$. Then

$$
\widetilde{\mathcal{P}}_{-}(X)=\left\{f \in \widetilde{\mathcal{P}}(X): f(D) T_{X} \text { is continuous in } \Lambda\right\} .
$$

Remark 21. Theorem 2 has no obvious generalisation to the general case. Consider the list $X=(1, a)$ for $a \in \mathbb{Z}$ and $a \geq 2$. Then $\left.B_{X}\right|_{[1, a]}=\frac{1}{a}$ and the function is linear with slope $\pm \frac{1}{a}$ on $[0,1]$ and $[a, a+1]$.

$$
\widetilde{\mathcal{P}}(1, a)=\operatorname{span}\left\{1, x, e_{2 \pi i / a} x, \ldots, e_{2 \pi i(a-1) / a} x\right\} .
$$

The space $\widetilde{\mathcal{P}}(1, a)$ is $a+1$ dimensional, but all but one basis element (1) send $B_{X}$ to a function that is zero everywhere on $\{1, \ldots, a-1\}$ except in one point (which one depends on whether we use a limit from the left or the right). Hence there is no subspace of $\widetilde{\mathcal{P}}(X)$ that contains unique interpolants. 


\section{Arithmetic matroids and periodic $\mathcal{P}$-spaces}

Recall that the Hilbert series of the spaces $\mathcal{P}(X)$ and $\mathcal{P}_{-}(X)$ are specialisations of the Tutte polynomial of the matroid defined by $X$ (cf. $(8)$ ) and that $\mathcal{P}(X)$ has a 'matroid-theoretic' basis (Proposition 10). In this section we give analogues of these two statements that relate the periodic $\mathcal{P}$-spaces and the arithmetic matroid defined by $X$.

An arithmetic matroid is a pair $(\mathfrak{M}, m)$, where $\mathfrak{M}$ is a matroid on the ground set $A$ and $m: 2^{A} \rightarrow \mathbb{Z}_{\geq 0}$ is a function that satisfies certain axioms [7, 20]. The function $m$ is called the multiplicity function. The prototype of an arithmetic matroid is the one that is canonically associated with a finite list of vectors $X$ in some lattice. In this case, for $S \subseteq X, m(S):=\left|(\operatorname{span}(S) \cap \Lambda) / \operatorname{span}_{\mathbb{Z}}(S)\right|$, where $\operatorname{span}_{\mathbb{Z}}(S)$ denotes the lattice generated by $S$. Note that if $S \subseteq X$ is linearly independent, then $m(S)$ is equal to the number of lattice points in the half-open parallelepiped $\left\{\sum_{s \in S} \lambda_{s} s: 0 \leq \lambda_{s}<1\right\}$.

The arithmetic Tutte polynomial [7,20] is defined as

$$
M_{X}(x, y)=\sum_{S \subseteq X} m(S)(x-1)^{\mathrm{rk}(X)-\operatorname{rk}(S)}(y-1)^{|S|-\mathrm{rk}(S)} .
$$

Note that there is a natural decomposition $\widetilde{\mathcal{P}}(X)=\bigoplus_{i \geq 0} \bigoplus_{\phi \in \mathcal{V}(X)} e_{\phi} P_{i, \phi}$, where each of the spaces $P_{i, \phi}$ contains only homogeneous polynomials of degree $i$. This allows us to define the Hilbert series $\operatorname{Hilb}(\widetilde{\mathcal{P}}(X), q)=\sum_{i \geq 0}\left(\sum_{\phi \in \mathcal{V}(X)} \operatorname{dim} P_{i, \phi}\right) q^{i}$

Proposition 22. Let $X \subseteq \Lambda \subseteq U \cong \mathbb{R}^{d}$ be a finite list of vectors that spans $U$. Then

$$
\operatorname{Hilb}(\widetilde{\mathcal{P}}(X), q)=q^{|(x \in X: x \neq 0)|-d} M_{X}\left(1, \frac{1}{q}\right) .
$$

Proposition 23 (Homogeneous basis). Let $X \subseteq \Lambda \subseteq U \cong \mathbb{R}^{d}$ be a finite list of vectors that spans $U$. Then the set $\widetilde{\mathcal{B}}(X):=\left\{e_{\phi} p_{X \backslash\left(B \cup\left(E(B) \cap X_{\phi}\right)\right)}: B \in \mathbb{B}(X), \phi \in \mathcal{V}(B)\right\}$ is a basis for $\widetilde{\mathcal{P}}(X)$ formed by homogeneous quasi-polynomials. Here $E(B)$ denotes the set of externally active elements in $X$ with respect to the basis $B$.

Proposition 24 (Inhomogeneous basis). Let $X \subseteq \Lambda \subseteq U \cong \mathbb{R}^{d}$ be a finite list of vectors that spans $U$. Let $w$ be a short affine regular vector. Then $\left\{f_{z}: z \in \overline{\mathcal{Z}}(X, w)\right\}$ is a basis for $\widetilde{\mathcal{P}}(X)$.

Remark 25. The internal periodic $\mathcal{P}$-space $\widetilde{\mathcal{P}}_{-}(X)$ is a subspace of $\widetilde{\mathcal{P}}(X)$ that satisfies $\operatorname{dim} \widetilde{\mathcal{P}}_{-}(X)=$ $\left|\mathcal{Z}_{-}(X)\right|=M_{X}(0,1)$ and it is the subspace of $\widetilde{\mathcal{P}}(X)$ whose elements leave $T_{X}$ continuous. Hence it shares many properties with $\mathcal{P}_{-}(X)$. It would be nice to show that it is homogeneous with Hilbert series $q^{N-d} M_{X}\left(1, q^{-1}\right)$ and to find another description of it. The author is currently working on these problems.

\section{Examples}

Example 26. If $X$ is not unimodular, then $\psi_{X}(\operatorname{Todd}(X, z))$ is in general not contained in $\mathcal{P}_{-}(X)$. Consider for example $X=(2,1)$ and $\operatorname{Todd}(X, z)=\frac{2 x}{e^{2 x}-1} \frac{x}{1-e^{-x}}$. Then

$$
\psi_{X}\left(f_{z}\right)=\psi_{X}\left(2\left(1+2 B_{1} x\right)\left(1-B_{1} x\right)\right)=2-x \notin \mathcal{P}_{-}(X)=\mathbb{R}
$$



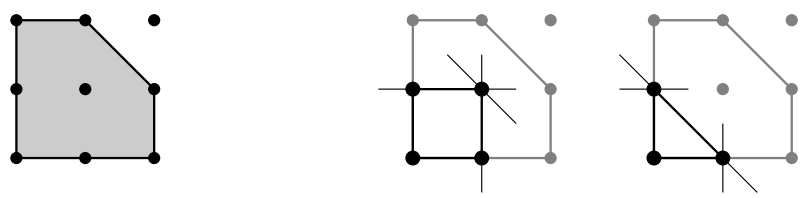

Figure 1: The pentagon $\square$, square $\square$, and triangle $\Delta$ that we discuss in Example 28

Example 27. Let $X=((1,0),(0,1),(1,1)) \subseteq \mathbb{Z}^{2}$. Then $\mathcal{P}_{-}(X)=\mathbb{R}, \mathcal{P}(X)=\operatorname{span}\left\{1, s_{1}, s_{2}\right\}$, $\mathcal{Z}_{-}(X)=\{(1,1)\}$, and $f_{(1,1)}=1 . \Pi_{X}\left(u_{1}, u_{2}\right) \cong\left[0, \min \left(u_{1}, u_{2}\right)\right] \subseteq \mathbb{R}^{1}$. The multivariate spline and the vector partition function are

$$
\begin{gathered}
T_{X}\left(u_{1}, u_{2}\right)=\left\{\begin{array}{l}
u_{2} \text { for } 0 \leq u_{2} \leq u_{1} \\
u_{1} \text { for } 0 \leq u_{1} \leq u_{2}
\end{array}\right. \\
\text { and } \mathcal{T}_{X}\left(u_{1}, u_{2}\right)= \begin{cases}u_{2}+1 & \text { for } 0 \leq u_{2} \leq u_{1} \\
u_{1}+1 & \text { for } 0 \leq u_{1} \leq u_{2}\end{cases}
\end{gathered}
$$

Corollary 6 correctly predicts that $\left.T_{X}(u)\right|_{\mathbb{Z}^{2}}=\mathcal{T}_{X}(u-(1,1))$. The figure on the right shows the six non-zero local pieces of $B_{X}$ and the seven polynomials $f_{z}$ attached to the lattice points of the zonotope $Z(X)$.

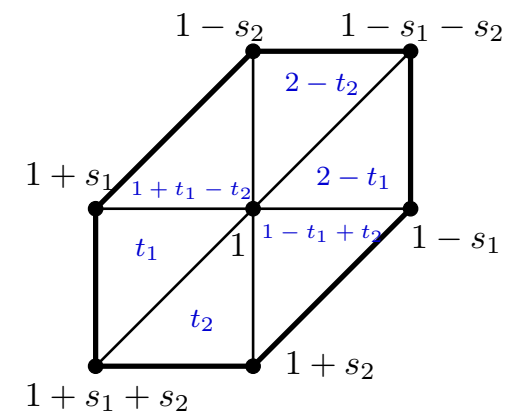

Example 28. We consider the polygons in Figure 1, which we will denote by $\square, \square$, and $\Delta$. These polytopes are defined by the matrix

$$
X=\left[\begin{array}{lllll}
1 & 0 & 0 & 1 & 0 \\
0 & 1 & 0 & 0 & 1 \\
0 & 0 & 1 & 1 & 1
\end{array}\right],
$$

where the first three columns correspond to slack variables and the rows of the last two are the normal vectors of the movable facets. The corresponding zonotope has two interior lattice points: $(1,1,1)$ corresponds to $\square$ and $(1,1,2)$ to $\Delta$.

The projections of the Todd operators are $f_{\square}=1+s_{3} / 2$ and $f_{\Delta}=1-s_{3} / 2$, where $s_{3}$ corresponds to shifting the diagonal face of the pentagon. Elementary calculations show that shifting this face outward ${ }^{(i)}$ by $\varepsilon \in[-1,1]$ increases the volume of the pentagon by $\left(\varepsilon-\frac{1}{2} \varepsilon^{2}\right)$. This implies $\frac{\partial}{\partial s_{3}} \operatorname{vol}($ pentagon $)=1$.

The volume of the pentagon is 3.5 . Corollary 6 correctly predicts that

$$
\left|\square \cap \mathbb{Z}^{2}\right|=\operatorname{vol}(\bullet)+\frac{1}{2} \frac{\partial}{\partial s_{3}} \operatorname{vol}(\bullet)=4 \quad \text { and } \quad\left|\Delta \cap \mathbb{Z}^{2}\right|=\operatorname{vol}(\bullet)-\frac{1}{2} \frac{\partial}{\partial s_{3}} \operatorname{vol}(\bullet)=3 .
$$

The projection to $\mathcal{P}(X)$ of the unshifted Todd operator $\operatorname{Todd}(X, 0)$ is a lot more complicated: $f_{\square}=$ $1+s_{1}+s_{2}+\frac{3}{2} s_{3}+s_{1} s_{2}+s_{1} s_{3}+s_{2} s_{3}+s_{3}^{2}$.

Since $\mathcal{Z}_{-}(X)$ contains only two points, the box spline $B_{X}$ must assume the value $\frac{1}{2}$ at both points. 13 correctly predicts that the volume of $\square$ is the arithmetic mean of the number of integer points in $\square$ and $\Delta$.

(i) This means replacing the inequality $a+b \leq 3$ by $a+b \leq 3+\varepsilon$. 


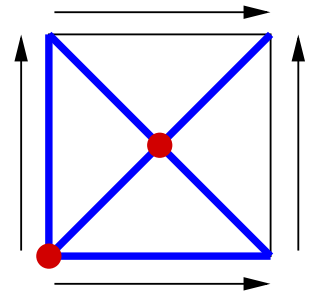

(a) The toric arrangement in $\mathbb{R}^{2} / \mathbb{Z}^{2}$

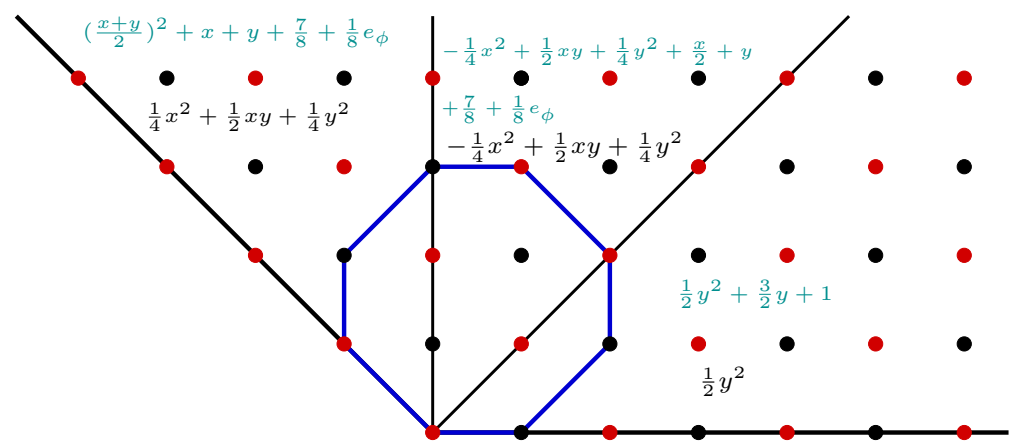

(b) The spline and the vector partition function have three local pieces each.

Figure 2: The multivariate spline, vector partition function, and toric arrangement corresponding to the Zwart-Powell element (cf. Example 29,

Example 29. We consider the matrix

$$
\begin{aligned}
X=\left(\begin{array}{cccc}
1 & 0 & 1 & -1 \\
0 & 1 & 1 & 1
\end{array}\right) . & \widetilde{\mathcal{P}}(X)=\operatorname{span}\left\{1, s_{1}, s_{2}, s_{1} s_{2}, s_{1}^{2}, s_{2}^{2}, e_{\phi} s_{1} s_{2}\right\} \\
\phi:=(1 / 2,1 / 2) \in(\mathbb{R} / \mathbb{Z})^{2} & \widetilde{\mathcal{B}}(X)=\left\{1, s_{2}, s_{2}\left(s_{1}+s_{2}\right), s_{1}, s_{1}\left(s_{1}+s_{2}\right), s_{1} s_{2}, e_{\phi} s_{1} s_{2}\right\}
\end{aligned}
$$

The corresponding box spline is known in the literature as the Zwart-Powell element.

$$
\begin{array}{ll}
f_{(0,1)}=1+\frac{1}{2} s_{1}+\frac{1}{2} s_{2}+\frac{1}{4} s_{1} s_{2}-e_{\phi} \frac{s_{1} s_{2}}{4} & f_{(1,1)}=1-\frac{1}{2} s_{1}+\frac{1}{2} s_{2}-\frac{1}{4} s_{1} s_{2}+e_{\phi} \frac{s_{1} s_{2}}{4} \\
f_{(0,2)}=1+\frac{1}{2} s_{1}-\frac{1}{2} s_{2}-\frac{1}{4} s_{1} s_{2}+e_{\phi} \frac{s_{1} s_{2}}{4} & f_{(1,2)}=1-\frac{1}{2} s_{1}-\frac{1}{2} s_{2}+\frac{1}{4} s_{1} s_{2}-e_{\phi} \frac{s_{1} s_{2}}{4}
\end{array}
$$

The internal space is $\widetilde{\mathcal{P}}_{-}(X)=\operatorname{span}\left\{1, s_{1}, s_{2},\left(1-e_{\phi}\right) s_{1} s_{2}\right\}$. The arithmetic Tutte polynomial is $M_{X}(x, y)=x^{2}+y^{2}+2 x+2 y+1$. Furthermore, $q^{2} M_{X}\left(1, q^{-1}\right)=1+2 q+4 q^{2}=\operatorname{Hilb}(\widetilde{\mathcal{P}}(X, q)$ and $q^{2} M_{X}\left(0, q^{-1}\right)=1+2 q+q^{2}=\operatorname{Hilb}\left(\widetilde{\mathcal{P}}_{-}(X), q\right)$.

\section{References}

[1] A. A. Akopyan and A. A. Saakyan, A system of differential equations that is related to the polynomial class of translates of a box spline, Mat. Zametki 44 (1988), no. 6, 705-724, 861.

[2] Federico Ardila and Alexander Postnikov, Combinatorics and geometry of power ideals, Trans. Amer. Math. Soc. 362 (2010), no. 8, 4357-4384.

[3] _ Two counterexamples for power ideals of hyperplane arrangements, 2012, arXiv:1211. 1368, to appear in Trans. Amer. Math. Soc. as a correction to [2].

[4] Matthias Beck and Sinai Robins, Computing the continuous discretely, Undergraduate Texts in Mathematics, Springer, New York, 2007, Integer-point enumeration in polyhedra. 
[5] Michel Brion and Michèle Vergne, Residue formulae, vector partition functions and lattice points in rational polytopes, J. Amer. Math. Soc. 10 (1997), no. 4, 797-833.

[6] David A. Cox, John B. Little, and Henry K. Schenck, Toric varieties, Graduate Studies in Mathematics, vol. 124, American Mathematical Society, Providence, RI, 2011.

[7] Michele D'Adderio and Luca Moci, Arithmetic matroids, the Tutte polynomial and toric arrangements, Adv. Math. 232 (2013), no. 1, 335-367.

[8] Carl de Boor, Nira Dyn, and Amos Ron, On two polynomial spaces associated with a box spline, Pacific J. Math. 147 (1991), no. 2, 249-267.

[9] Carl de Boor, Klaus Höllig, and Sherman D. Riemenschneider, Box splines, Applied Mathematical Sciences, vol. 98, Springer-Verlag, New York, 1993.

[10] Corrado De Concini and Claudio Procesi, Topics in hyperplane arrangements, polytopes and boxsplines, Universitext, Springer, New York, 2011.

[11] Corrado De Concini, Claudio Procesi, and Michèle Vergne, Box splines and the equivariant index theorem, J. Inst. Math. Jussieu 12 (2013), 503-544.

[12] Nira Dyn and Amos Ron, Local approximation by certain spaces of exponential polynomials, approximation order of exponential box splines, and related interpolation problems, Trans. Amer. Math. Soc. 319 (1990), no. 1, 381-403.

[13] Friedrich Hirzebruch, Neue topologische Methoden in der algebraischen Geometrie, Ergebnisse der Mathematik und ihrer Grenzgebiete (N.F.), Heft 9, Springer-Verlag, Berlin, 1956.

[14] Olga Holtz and Amos Ron, Zonotopal algebra, Adv. Math. 227 (2011), no. 2, 847-894.

[15] Askold Khovanskiĭ and Aleksandr Pukhlikov, The Riemann-Roch theorem for integrals and sums of quasipolynomials on virtual polytopes, Algebra i Analiz 4 (1992), no. 4, 188-216.

[16] Matthias Lenz, Hierarchical zonotopal power ideals, European J. Combin. 33 (2012), no. 6.

[17] __ Zonotopal algebra and forward exchange matroids, 2012, 27 pages, arXiv:1204. $3869 \mathrm{v} 2$.

[18] _ Lattice points in polytopes, box splines, and Todd operators, 2013, 15 pages, arXiv: 1305.2784

[19] __ Interpolation, box splines, and lattice points in zonotopes, International Mathematics Research Notices (first published online July 10, 2013), 16 pages.

[20] Luca Moci, A Tutte polynomial for toric arrangements, Trans. Amer. Math. Soc. 364 (2012), no. 2.

[21] Michèle Vergne, Residue formulae for Verlinde sums, and for number of integral points in convex rational polytopes, European women in mathematics (Malta, 2001), World Sci. Publ., River Edge, NJ, 2003, pp. 225-285. 